\title{
Kinetics and mechanism of the oxidation of some diols by benzyltrimethylammonium tribromide
}

\author{
GARIMA GOSWAMI, SEEMA KOTHARI and KALYAN K BANERJI* \\ Department of Chemistry, JNV University, Jodhpur 342 005, India \\ e-mail: kkbanerji@usa.net
}

MS received 16 August 2000; revised 8 December 2000

\begin{abstract}
The kinetics of oxidation of five vicinal and four non-vicinal diols, and two of their monoethers by benzyltrimethylammonium tribromide (BTMAB) have been studied in 3:7 $(v / v)$ acetic acid-water mixture. The vicinal diols yield the carbonyl compounds arising out of the glycol bond fission while the other diols give the hydroxycarbonyl compounds. The reaction is first-order with respect to BTMAB. Michaelis-Menten type kinetics is observed with respect to diol. Addition of benzyltrimethylammonium chloride does not affect the rate. Tribromide ion is postulated to be the reactive oxidizing species. Oxidation of $\left[1,1,2,2{ }^{2} \mathrm{H}_{4}\right]$ ethanediol shows the absence of a kinetic isotope effect. The reaction exhibits substantial solvent isotope effect. A mechanism involving a glycol-bond fission has been proposed for the oxidation of the vicinal diols. The other diols are oxidized by a hydride ion transfer to the oxidant, as are the monohydric alcohols.
\end{abstract}

Keywords. Diols; benzyltrimethylammonium tribromide; oxidation; kinetics; mechanism; kinetic isotope effect.

\section{Introduction}

Benzyltrimethylammonium tribromide (BTMAB) has been used as an effective halogenating and oxidizing agent in synthetic organic chemistry ${ }^{1-3}$. We are interested in kinetic and mechanistic studies of oxidations by polyhalide ions, and several reports on the oxidation by $\mathrm{BTMAB}^{4-8}$, including those of monohydric alcohols, have emanated from our laboratory. There seems to be no report in the literature on the oxidation of diols by BTMAB. However, several oxidations of monohydric and polyhydric alcohols are known to follow different mechanistic pathways, e.g. oxidations by lead (IV) ${ }^{9}$, periodic acid $^{10}$, acid permanganate ${ }^{11}$ and bromamine- $\mathrm{B}^{12}$. Therefore, in this work, we study the oxidation of several diols by BTMAB in 3:7 ( $/ / v)$ acetic acid-water mixture, and discuss the mechanistic aspects.

\section{Experimental}

\subsection{Materials}

The diols and the monoethers (BDH or Fluka) were distilled under reduced pressure before use. BTMAB was prepared by the reported method ${ }^{1}$ and its purity was checked by

\footnotetext{
*For correspondence
} 
an iodometric method. [1,1,2,2- $\left.{ }^{2} \mathrm{H}_{4}\right]$ Ethanediol (DED) was prepared by reducing diethyl oxalate with lithium aluminium deuteride ${ }^{13}$. Its isotopic purity, determined by its ${ }^{1} \mathrm{H}$ NMR spectrum, was $91 \pm 5 \%$. Acetic acid was refluxed with chromic oxide and acetic anhydride for $6 \mathrm{~h}$ and then fractionated.

\subsection{Product analysis}

Product analysis was carried out under kinetic conditions. In a typical experiment, the diol $(0.1 \mathrm{~mol}), \mathrm{KBr}(3.6 \mathrm{~g}, 0.03 \mathrm{~mol})$ and BTMAB $(3.9 \mathrm{~g}, 0.01 \mathrm{~mol})$ were taken in 3:7 $(v / v)$ acetic acid-water solution $(100 \mathrm{ml})$ and the mixture was allowed to stand in the dark for $\approx 10 \mathrm{~h}$ to ensure completion of the reaction. It was then treated overnight with an excess $(250 \mathrm{ml})$ of saturated solution of 2,4-dinitrophenylhydrazine in $2 \mathrm{~mol} \mathrm{dm}^{-3} \mathrm{HCl}$. The precipitated 2,4-dinitrophenylhydrazone (DNP) was filtered off, dried, recrystallized from ethanol and weighed. The yield of DNP after recrystallization, in the case of ethanediol, was $3.65 \mathrm{~g}(87 \%)$. In other cases, the yields varied between 81 and $92 \%$. The DNP derivatives were found to be homogeneous by TLC except in the oxidation of propane-1,2- and butane-1,2-diols. In these cases the mixtures were separated by fractional crystallization. The identity of products was established by comparing the melting points of the DNP derivatives with the literature values ${ }^{14}$. In the oxidation of ethanediol, propane-1,2-diol and butane-1,2-, -2,3-, -1,3-, and -1,4-diols, the identity of the products was confirmed by using mixed melting points with authentic samples of DNP of formaldehyde, acetaldehyde, propionaldehyde, acetaldehyde, 3-hydroxybutanal and 4-hydroxybutanal respectively. The melting points were measured in open capillaries and are uncorrected. The results are summarized in table 1.

\subsection{Spectral studies}

UV-VIS spectra of (A) BTMAB $\left(0.001 \mathrm{~mol} \mathrm{dm}^{-3}\right)$ alone and (B)-(D) of propane-1,2-diol $\left(0 \cdot 005-0.05 \mathrm{~mol} \mathrm{dm}^{-3}\right)+$ BTMAB $\left(0.001 \mathrm{~mol} \mathrm{dm}^{-3}\right)$ were recorded on an HP diode-array spectrophotometer (Model 8452A) with a scanning speed of $600 \mathrm{~nm} \mathrm{~s}^{-1}$. The solvent was $3: 7(v / v)$ acetic acid-water and temperature was $285 \pm 1 \mathrm{~K}$. Spectra of the reaction

Table 1. Analysis of products in the oxidation of diols by BTMAB.

\begin{tabular}{llcc}
\hline Diol & \multicolumn{1}{c}{ Product } & m.p. of DNP $\left({ }^{\circ} \mathrm{C}\right)$ & Yield $^{\text {a }}(\%)$ \\
\hline Ethanediol & HCHO & 166 & 92 \\
Propane-1,2-diol & MeCHO, HCHO & 147,165 & 93 \\
Butane-2,3-diol & MeCHO & 145 & 91 \\
Butane-1,2-diol & EtCHO, HCHO & 143,164 & 94 \\
Pinacol & MeCOMe & 128 & 93 \\
Propane-1,3-diol & $\mathrm{HOCH}_{2} \mathrm{CH}_{2} \mathrm{CHO}$ & 131 & 90 \\
Butane-1,3-diol & ${\mathrm{MeCH}\left(\mathrm{OH}^{2} \mathrm{CH}_{2} \mathrm{CHO}\right.}_{\text {Butane-1,4-diol }}^{\mathrm{HOCH}_{2} \mathrm{CH}_{2} \mathrm{CH}_{2} \mathrm{CHO}}$ & 92 & 90 \\
Pentane-1,5-diol & $\mathrm{HOCH}_{2}\left(\mathrm{CH}_{2}\right)_{3} \mathrm{CHO}$ & 78 & 85 \\
3-Methoxybutan-1-ol & ${\mathrm{MeCH}\left(\mathrm{OMe}_{2} \mathrm{CHO}\right.}_{2} \mathrm{CHO}$ & 103 & 89 \\
2-Methoxyethanol & $\mathrm{MeOCH}_{2} \mathrm{CHO}$ & $118^{\mathrm{b}}$ & 91 \\
\hline
\end{tabular}

${ }^{a}$ Yield is of DNP derivative after recrystallization; ${ }^{b}$ instead of DNP, 4-nitrophenylhydrazone derivative was prepared 
mixtures (B) were also recorded at different times ranging from 30 to $900 \mathrm{~s}$. For (A) the blank was the solvent; for (B)-(D) the blank was a solution of propane-1,2-diol (0.005$\left.0.05 \mathrm{~mol} \mathrm{dm}^{-3}\right)$ in aqueous acetic acid. The time gap between the preparation of the reaction mixture and recording of spectra (B)-(D) was $<10 \mathrm{~s}$.

\subsection{Kinetic measurements}

The reactions were studied under pseudo-first-order conditions by taking an excess $(\times 15$ or greater) of the diol over BTMAB. The solvent was 3:7 (v/v) acetic acid-water, unless mentioned otherwise. Tribromide ion is known to dissociate into bromine and bromide ion. To suppress the dissociation, the reactions were carried out in the presence of an excess $\left(0.2 \mathrm{~mol} \mathrm{dm}{ }^{-3}\right)$ of potassium bromide. The reactions were studied at constant temperature $( \pm 0 \cdot 1 \mathrm{~K})$ and were followed by monitoring the decrease in the concentration of BTMAB at $354 \mathrm{~nm}$ for up to $80 \%$ reaction. Pseudo-first-order rate constants, $k_{\mathrm{obs}}$, were evaluated from linear plots $(r>0.995)$ of $\log [\mathrm{BTMAB}]$ against time. Duplicate kinetic runs showed that the rate constants are reproducible to within $\pm 3 \%$. Preliminary experiments showed that the reaction is not sensitive to changes in the ionic strength. Hence no attempt was made to keep the ionic strength constant.

\section{Results}

\subsection{Stoichiometry}

The oxidation of vicinal diols by BTMAB yields products arising out of glycol-bond fission, i.e. rupture of the bond between the carbon atoms bearing the hydroxy groups, while the other diols give products by simple oxidation of one of the hydroxy groups. Analyses of products indicate the following overall reactions.

$$
\begin{aligned}
& \mathrm{R}_{2} \mathrm{C}-\mathrm{CR}_{2} \mathrm{CH}_{\mathrm{OH}}+\mathrm{PhCH}_{2} \mathrm{Me}_{3} \mathrm{NBr}_{3} \rightarrow 2 \mathrm{R}_{2} \mathrm{C}=\mathrm{O}+\mathrm{PhCH}_{2} \mathrm{Me}_{3} \mathrm{~N}^{+} \mathrm{Br}^{-}+2 \mathrm{HBr}, \\
& \mathrm{HO}-\mathrm{CH}_{2}-\left(\mathrm{CH}_{2}\right)_{n}-\mathrm{CH}_{2} \mathrm{OH}+\mathrm{PhCH}_{2} \mathrm{Me}_{3} \mathrm{NBr}_{3} \rightarrow \\
& \quad \mathrm{HO}-\mathrm{CH}_{2}-\left(\mathrm{CH}_{2}\right)_{n}-\mathrm{CHO}+2 \mathrm{HBr}+\mathrm{PhCH}_{2} \mathrm{Me}_{3} \mathrm{~N}^{+} \mathrm{Br}^{-} .
\end{aligned}
$$

\subsection{Induced polymerization of acrylonitrile}

The oxidation of diols by BTMAB, in nitrogen atmosphere, failed to induce the polymerization of acrylonitrile. Further, the addition of acrylonitrile had no effect on the rate (table 2 ).

\subsection{Rate laws}

The reactions are first-order with respect to BTMAB. Further, the values of $k_{\mathrm{obs}}$ are independent of the initial concentration of BTMAB. The order with respect to diol is less than one (table 2). A plot of $1 / k_{\text {obs }}$ versus $1 /[$ diol] is linear with an intercept on the rate ordinate. Thus Michaelis-Menten type of kinetics is observed with respect to diols. This leads to the postulation of the following overall mechanism and rate law, 


$$
\begin{aligned}
& \text { diol }+ \text { oxidant } \stackrel{K}{\leftrightharpoons} \text { [complex }], \\
& {[\text { complex }] \stackrel{k_{2}}{\longrightarrow} \text { products, }} \\
& \text { rate }=k_{2} K[\text { diol] [oxidant }] /(1+K[\text { diol }]) .
\end{aligned}
$$

The dependence on the concentration of the diol was studied at different temperatures and the values of $K$ and $k_{2}$ were calculated from the double reciprocal plots. The thermodynamic parameters of the formation of the intermediate and the activation parameters of its decomposition were calculated from the values of $K$ and $k_{2}$ respectively at different temperatures (tables 3 and 4).

\subsection{Spectral studies}

A perusal of the spectra of BTMAB and BTMAB + propane-1,2-diol, at three different concentrations of the diol, showed that there is distinct increase in the absorbance of BTMAB on addition of diol which increases with increase in the concentration of diol (figure 1). The absorbance of the reaction mixture decreases with time. This supports the formation of an intermediate pre-equilibrium complex and its subsequent decomposition to the ultimate products.

\subsection{Effect of benzyltrimethylammonium ion}

Addition of benzyltrimethylammonium chloride has no effect on the oxidation of diol (table 5).

\begin{tabular}{|c|c|c|c|}
\hline \multirow{2}{*}{$\begin{array}{l}10^{3}[\text { BTMAB }] \\
\left(\mathrm{mol} \mathrm{dm}^{-3}\right)\end{array}$} & \multirow{2}{*}{$\begin{array}{c}{[\text { Diol }]} \\
\left(\mathrm{mol} \mathrm{dm}^{-3}\right)\end{array}$} & \multicolumn{2}{|c|}{$10^{5} k_{\mathrm{obs}}\left(\mathrm{s}^{-1}\right)$} \\
\hline & & Ethanediol & Propane-1,3-diol \\
\hline $1 \cdot 0$ & 0.05 & $5 \cdot 48$ & 34.0 \\
\hline $1 \cdot 0$ & $0 \cdot 10$ & 9.94 & 59.9 \\
\hline $1 \cdot 0$ & $0 \cdot 20$ & $16 \cdot 9$ & 101 \\
\hline $1 \cdot 0$ & $0 \cdot 30$ & $23 \cdot 5$ & 135 \\
\hline $1 \cdot 0$ & 0.50 & $28 \cdot 5$ & 164 \\
\hline $1 \cdot 0$ & $0 \cdot 80$ & $35 \cdot 7$ & 200 \\
\hline $1 \cdot 0$ & $1 \cdot 50$ & $42 \cdot 5$ & 235 \\
\hline $0 \cdot 5$ & $0 \cdot 50$ & $28 \cdot 1$ & 170 \\
\hline $2 \cdot 0$ & $0 \cdot 50$ & $28 \cdot 7$ & 161 \\
\hline $3 \cdot 0$ & $0 \cdot 50$ & $29 \cdot 1$ & 168 \\
\hline $5 \cdot 0$ & $0 \cdot 50$ & $28 \cdot 4$ & 160 \\
\hline $1 \cdot 0$ & $0 \cdot 50$ & $28 \cdot 0^{*}$ & $166 *$ \\
\hline
\end{tabular}

Table 2. Rate constants for the oxidation of ethanediol and propane-1,3-diol by BTMAB at $313 \mathrm{~K}$.

*Contained 0.005 mol dm ${ }^{-3}$ acrylonitrile 
Table 3. Formation constants and thermodynamic parameters of the diol-oxidant complexes.

\begin{tabular}{|c|c|c|c|c|c|c|c|}
\hline \multirow[b]{2}{*}{ Diol } & \multicolumn{4}{|c|}{$K\left(\mathrm{dm}^{-3} \mathrm{~mol}^{-1}\right)$ at } & \multirow{2}{*}{$\begin{array}{c}\Delta H \\
\left(\mathrm{~kJ} \mathrm{~mol}^{-1}\right)\end{array}$} & \multirow{2}{*}{$\begin{array}{c}\Delta S \\
\left(\mathrm{~J} \mathrm{~K}^{-1} \mathrm{~mol}^{-1}\right)\end{array}$} & \multirow{2}{*}{$\begin{array}{c}\Delta G \\
\left(\mathrm{~kJ} \mathrm{~mol}^{-1}\right)\end{array}$} \\
\hline & $293 \mathrm{~K}$ & $303 \mathrm{~K}$ & $313 \mathrm{~K}$ & $323 \mathrm{~K}$ & & & \\
\hline Ethane-1,2-diol & 3.79 & 2.95 & $2 \cdot 13$ & $1 \cdot 59$ & $-25 \cdot 6 \pm 0.8$ & $-68 \pm 3$ & $-5.47 \pm 0.6$ \\
\hline Propane-1,2-diol & $4 \cdot 20$ & $3 \cdot 06$ & $2 \cdot 30$ & $1 \cdot 68$ & $-26 \cdot 3 \pm 0 \cdot 4$ & $-70 \pm 1$ & $-5 \cdot 66 \pm 0 \cdot 3$ \\
\hline Butane-2,3-diol & $5 \cdot 51$ & $4 \cdot 15$ & $3 \cdot 21$ & $2 \cdot 47$ & $-23.4 \pm 0.2$ & $-58 \pm 1$ & $-6 \cdot 37 \pm 0.1$ \\
\hline Butane-1,2-diol & $4 \cdot 01$ & 2.99 & $2 \cdot 19$ & 1.76 & $-24.4 \pm 0.5$ & $-64 \pm 2$ & $-5 \cdot 56 \pm 0.4$ \\
\hline Pinacol & 4.85 & 3.69 & $2 \cdot 88$ & $2 \cdot 29$ & $-22 \cdot 2 \pm 0.1$ & $-55 \pm 1$ & $-6 \cdot 07 \pm 0.1$ \\
\hline Propane-1,3-diol & $6 \cdot 05$ & $4 \cdot 11$ & $2 \cdot 82$ & 1.90 & $-32 \cdot 8 \pm 0.5$ & $-50 \pm 2$ & $-5 \cdot 62 \pm 0.4$ \\
\hline Butane-1,3-diol & $6 \cdot 15$ & $4 \cdot 39$ & $3 \cdot 11$ & $2 \cdot 27$ & $-28 \cdot 7 \pm 0 \cdot 2$ & $-75 \pm 1$ & $-6 \cdot 57 \pm 0 \cdot 2$ \\
\hline Butane-1,4-diol & $5 \cdot 89$ & $4 \cdot 30$ & $3 \cdot 20$ & $2 \cdot 50$ & $-25 \cdot 1 \pm 0 \cdot 3$ & $-63 \pm 1$ & $-6.49 \pm 0.2$ \\
\hline Pentane-1,5-diol & $6 \cdot 27$ & $4 \cdot 61$ & $3 \cdot 41$ & $2 \cdot 46$ & $-26 \cdot 9 \pm 0.5$ & $-68 \pm 2$ & $-6 \cdot 66 \pm 0 \cdot 4$ \\
\hline 3-Methoxy-butan-1-ol & $7 \cdot 11$ & $5 \cdot 11$ & $3 \cdot 59$ & $2 \cdot 64$ & $-28.6 \pm 0.3$ & $-73 \pm 1$ & $-6.93 \pm 0.3$ \\
\hline 2-Methoxy-ethanol & $5 \cdot 92$ & $4 \cdot 00$ & $2 \cdot 63$ & $1 \cdot 78$ & $-34 \cdot 1 \pm 0 \cdot 5$ & $-94 \pm 2$ & $-6 \cdot 40 \pm 0.4$ \\
\hline $\mathrm{DED}^{\mathrm{a}}$ & $3 \cdot 81$ & $2 \cdot 89$ & $2 \cdot 17$ & $1 \cdot 60$ & $-25 \cdot 2 \pm 0.5$ & $-67 \pm 2$ & $-5.46 \pm 0.4$ \\
\hline
\end{tabular}

${ }^{\mathrm{a}}\left[1,1,2,2-{ }^{2} \mathrm{H}_{4}\right]$ ethanediol

Table 4. Rate constants and activation parameters of the decomposition of dioloxidant complexes.

\begin{tabular}{|c|c|c|c|c|c|c|c|}
\hline \multirow[b]{2}{*}{ Diol } & \multicolumn{4}{|c|}{$10^{2} k_{2}\left(\mathrm{~s}^{-1}\right)$ at } & \multirow{2}{*}{$\begin{array}{c}\Delta H^{*} \\
\left(\mathrm{~kJ} \mathrm{~mol}^{-1}\right)\end{array}$} & \multirow{2}{*}{$\begin{array}{c}\Delta S^{*} \\
\left(\mathrm{~J} \mathrm{~K}^{-1} \mathrm{~mol}^{-1}\right)\end{array}$} & \multirow{2}{*}{$\begin{array}{c}\Delta G^{*} \\
\left(\mathrm{~kJ} \mathrm{~mol}^{-1}\right)\end{array}$} \\
\hline & $293 \mathrm{~K}$ & $303 \mathrm{~K}$ & $313 \mathrm{~K}$ & $323 \mathrm{~K}$ & & & \\
\hline Ethane-1,2-diol & $1 \cdot 77$ & $3 \cdot 19$ & $5 \cdot 70$ & $9 \cdot 91$ & $42 \cdot 7 \pm 0 \cdot 4$ & $-172 \pm 1$ & $93.7 \pm 0.3$ \\
\hline Propane-1,2-diol & 2.53 & $4 \cdot 39$ & $7 \cdot 61$ & 12.7 & $39.9 \pm 0.3$ & $-178 \pm 1$ & $92 \cdot 8 \pm 0.2$ \\
\hline Butane-2,3-diol & $4 \cdot 70$ & $7 \cdot 80$ & $12 \cdot 9$ & $20 \cdot 8$ & $36 \cdot 5 \pm 0 \cdot 3$ & $-184 \pm 1$ & $91 \cdot 4 \pm 0 \cdot 2$ \\
\hline Butane-1,2-diol & $6 \cdot 82$ & $11 \cdot 1$ & $17 \cdot 9$ & $27 \cdot 6$ & $34 \cdot 2 \pm 0 \cdot 2$ & $-189 \pm 1$ & $90 \cdot 5 \pm 0 \cdot 1$ \\
\hline Pinacol & $7 \cdot 49$ & $12 \cdot 2$ & $19 \cdot 0$ & $28 \cdot 9$ & $32 \cdot 8 \pm 0 \cdot 2$ & $-193 \pm 1$ & $90 \cdot 2 \pm 0 \cdot 1$ \\
\hline Propane-1,3-diol & 1.71 & $4 \cdot 30$ & $11 \cdot 2$ & $29 \cdot 1$ & $71 \cdot 8 \pm 1 \cdot 3$ & $-73 \pm 4$ & $93 \cdot 3 \pm 1 \cdot 0$ \\
\hline Butane-1,3-diol & $2 \cdot 81$ & $7 \cdot 01$ & $17 \cdot 5$ & $44 \cdot 7$ & $69 \cdot 9 \pm 1 \cdot 2$ & $-75 \pm 4$ & $92 \cdot 1 \pm 0 \cdot 9$ \\
\hline Butane-1,4-diol & $2 \cdot 41$ & $6 \cdot 10$ & $15 \cdot 6$ & $38 \cdot 9$ & $70 \cdot 5 \pm 0 \cdot 9$ & $-74 \pm 3$ & $92.5 \pm 0.7$ \\
\hline Pentane-1,5-diol & $2 \cdot 98$ & $7 \cdot 54$ & $18 \cdot 7$ & $46 \cdot 8$ & $69 \cdot 6 \pm 0 \cdot 9$ & $-76 \pm 3$ & $92 \cdot 0 \pm 0.7$ \\
\hline 3-Methoxy-butan-1-ol & $3 \cdot 41$ & $8 \cdot 46$ & $21 \cdot 2$ & $52 \cdot 5$ & $69 \cdot 2 \pm 1 \cdot 0$ & $-76 \pm 3$ & $91.6 \pm 0.8$ \\
\hline 2-Methoxy-ethanol & $2 \cdot 00$ & $5 \cdot 20$ & $12 \cdot 5$ & $32 \cdot 9$ & $70 \cdot 4 \pm 1 \cdot 3$ & $-76 \pm 4$ & $92 \cdot 9 \pm 1 \cdot 0$ \\
\hline $\mathrm{DED}^{\mathrm{a}}$ & 1.71 & $3 \cdot 22$ & $5 \cdot 60$ & 9.86 & $43 \cdot 2 \pm 0 \cdot 3$ & $-170 \pm 1$ & $93.7 \pm 0.3$ \\
\hline
\end{tabular}

${ }^{\mathrm{a}}\left[1,1,2,2-{ }^{2} \mathrm{H}_{4}\right]$ ethanediol

Table 5. Effect of benzyltrimethylammonium chloride (BTMC) on the rate of oxidation of ethanediol by BTMAB.

$\left[\right.$ BTMAB] $=0.001 \mathrm{~mol} \mathrm{dm}^{-3},[\mathrm{diol}]=0.5 \mathrm{~mol} \mathrm{dm}^{-3}, T=313 \mathrm{~K}$

\begin{tabular}{lrrrrrr}
\hline $10^{3}[\mathrm{BTMC}]\left(\mathrm{mol} \mathrm{dm}^{-3}\right)$ & 0.0 & 1.0 & 2.0 & 4.0 & 6.0 & 8.0 \\
$10^{5} k_{\mathrm{obs}} / \mathrm{s}^{-1}$ & 28.5 & 28.0 & 28.9 & 28.2 & 27.6 & 29.1 \\
\hline
\end{tabular}




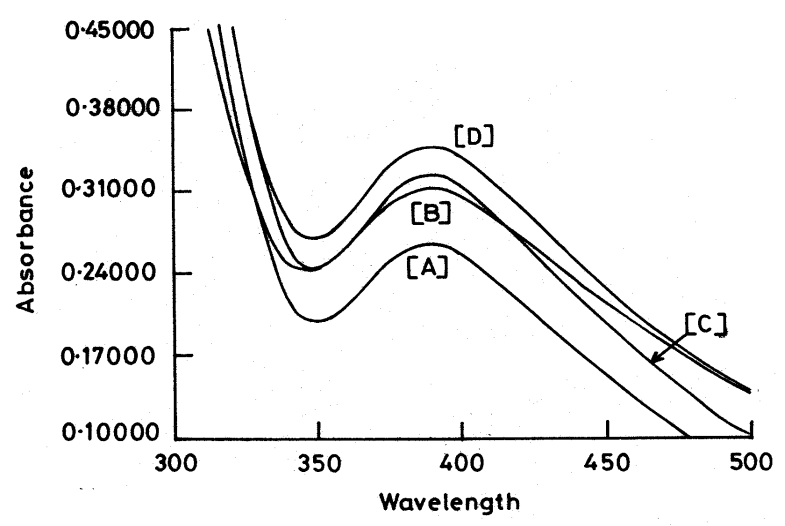

Figure 1. UV-VIS spectra of [A] $0.001 \mathrm{~mol} \mathrm{dm}^{-3} \mathrm{BTMAB},[\mathrm{B}] 0.001 \mathrm{~mol} \mathrm{dm}^{-3}$ $\mathrm{BTMAB}+0.005 \mathrm{~mol} \mathrm{dm}^{-3}$ propane-1,3-diol, $[\mathrm{C}] \quad 0.001 \mathrm{~mol} \mathrm{dm}^{-3}$ BTMAB + $0.01 \mathrm{~mol} \mathrm{dm}^{-3}$ propane-1,3-diol and [D] $0.001 \mathrm{~mol} \mathrm{dm}^{-3} \mathrm{BTMAB}+0.05 \mathrm{~mol} \mathrm{dm}^{-3}$ propane-1,3-diol. Solvent: 3:7 (v/v) acetic acid-water, temperature $285 \pm 1 \mathrm{~K}$.

\subsection{Solvent effect}

Rates of oxidation of diol were determined in solvents containing different amounts of acetic acid and water. It was observed that rate increases with increase in the amount of water in the solvent mixture.

To determine whether the changes in solvent composition affect the formation constant, $K$, and/or the rate of decomposition, $k_{2}$, the dependence on diol concentration was studied in solvents of different composition. Results showed that the rate constant for the decomposition of the complex, $k_{2}$, increases with increase in the polarity of the medium, however, the formation constant, $K$, decreases (table 6).

\subsection{Kinetic isotope effect}

To ascertain the importance of the cleavage of $\alpha-\mathrm{C}-\mathrm{H}$ bond in the rate-determining step, the oxidation of $\left[1,1,2,2-{ }^{2} \mathrm{H}_{4}\right]$ ethanediol was studied. The results showed that both the formation constant of the complex and the rate of its decomposition do not change on isotopic substitution (tables 3 and 4).

\subsection{Solvent isotope effect}

The rates of oxidation of ethanediol, propane-1,3-diol, and 3-methoxybutan-1-ol were obtained in deuterium oxide (overall $95 \% \mathrm{D}_{2} \mathrm{O}$ ). This set of experiments was carried out in aqueous solutions. It was observed that the value of the formation constant, $K$, of the complex is not sensitive to the isotopic composition of the solvent. However, the rate constant, $k_{2}$, of the disproportionation of the complex exhibits considerable change from $\mathrm{H}_{2} \mathrm{O}$ to $\mathrm{D}_{2} \mathrm{O}$ (table 7). 
Table 6. Dependence of rate on the concentration of ethanediol in the solvents of different compositions. $[\mathrm{BTMAB}]=0.001 \mathrm{~mol} \mathrm{dm}^{-3}, T=313 \mathrm{~K}$

\begin{tabular}{|c|c|c|c|c|c|c|}
\hline \multirow{2}{*}{$\begin{array}{l}{[\text { Diol] }} \\
\left(\mathrm{mol} \mathrm{dm}^{-3}\right)\end{array}$} & \multicolumn{6}{|c|}{$10^{5} k_{\mathrm{obs}} / \mathrm{s}^{-1}[$ at $\% \mathrm{AcOH}(v / v)]$} \\
\hline & 20 & 30 & 40 & 50 & 70 & 80 \\
\hline $0 \cdot 1$ & $12 \cdot 3$ & $9 \cdot 94$ & $10 \cdot 2$ & $4 \cdot 70$ & $4 \cdot 61$ & $3 \cdot 35$ \\
\hline 0.2 & 21.5 & $16 \cdot 9$ & $16 \cdot 5$ & $6 \cdot 91$ & $6 \cdot 30$ & 4.27 \\
\hline $0 \cdot 3$ & 28.5 & 23.5 & $20 \cdot 0$ & $8 \cdot 33$ & $7 \cdot 32$ & 4.81 \\
\hline 0.5 & 39.5 & 28.5 & 25.5 & 9.65 & $8 \cdot 10$ & $5 \cdot 11$ \\
\hline 0.8 & 49.0 & 35.7 & $29 \cdot 0$ & $10 \cdot 9$ & 8.81 & 6.58 \\
\hline $1 \cdot 5$ & $62 \cdot 0$ & $42 \cdot 5$ & $33 \cdot 8$ & $11 \cdot 5$ & $9 \cdot 29$ & $5 \cdot 71$ \\
\hline$K\left(\mathrm{dm}^{3} \mathrm{~mol}^{-1}\right)$ & 1.66 & $2 \cdot 13$ & 3.42 & $5 \cdot 57$ & 8.43 & $12 \cdot 4$ \\
\hline$k_{2}\left(\mathrm{~s}^{-1}\right)$ & $8 \cdot 64$ & $5 \cdot 70$ & 4.00 & $1 \cdot 31$ & 1.00 & 0.60 \\
\hline
\end{tabular}

Table 7. Solvent isotope effect in the oxidation of diols by BTMAB at $313 \mathrm{~K}$.

\begin{tabular}{lccc}
\hline Diol & $\begin{array}{c}K_{2}\left(\mathrm{H}_{2} \mathrm{O}\right) \\
\left(\mathrm{mol} \mathrm{dm}^{-3}\right)\end{array}$ & $\begin{array}{c}k_{2}\left(\mathrm{D}_{2} \mathrm{O}\right) \\
\left(\mathrm{mol} \mathrm{dm}^{-3}\right)\end{array}$ & $\begin{array}{c}k_{2}\left(\mathrm{H}_{2} \mathrm{O}\right) / \\
k_{2}\left(\mathrm{D}_{2} \mathrm{O}\right)\end{array}$ \\
\hline Ethanediol & $5 \cdot 70$ & $1 \cdot 16$ & 4.91 \\
Propane-1,3-diol & 29.9 & $12 \cdot 3$ & 2.43 \\
3-Methoxybutan-1-ol & $60 \cdot 0$ & 22.6 & 2.65 \\
\hline
\end{tabular}

\section{Discussion}

We have earlier carried out some conductivity measurements to determine the nature of BTMAB in aqueous acetic acid solution ${ }^{4}$. It was observed that acetic acid has very low conductivity. Addition of BTMAB increases the conductivity of acetic acid. We measured the conductivity of BTMAB in solvents containing different proportions of acetic acid (100-30\%) and water. We found that the conductivity increases sharply as the water content is increased initially, but reaches a limiting value in about $70 \%$ acetic acidwater mixture. Therefore, BTMAB can be considered an ionic compound that exists under our reaction conditions as benzytrimethylammonium and tribromide ions. No effect of added benzyltrimethylammonium ion (table 5) also indicates that the equilbrium (6) below, lies far towards the right,

$$
\mathrm{PhCH}_{2} \mathrm{Me}_{3} \mathrm{NBr}_{3} \leftrightharpoons \mathrm{PhCH}_{2} \mathrm{Me}_{3} \mathrm{~N}^{+}+\mathrm{Br}_{3}^{-}
$$

Tribromide ion is known to dissociate as follows,

$$
\mathrm{Br}_{3}^{-} \leftrightharpoons \mathrm{Br}_{2}+\mathrm{Br}^{-}
$$

The value of the equilibrium constant ${ }^{15}$, in $50 \%$ aqueous acetic acid, is $0.02 \mathrm{~mol} \mathrm{dm}^{-3}$. However, in the presence of a large excess of bromide ions, dissociation is likely to be suppressed. Therefore, the most likely reactive oxidizing species, in this reaction, is tribromide ion. 
Decrease in values of $k_{2}$ with increase in the amount of acetic acid indicates that increase in the solvent polarity facilitates the reaction. This can be explained if one assumes that in the rate-determining step the transition state is more polar than the reactant. A plot of $\log k_{2}$ against the inverse of relative permittivity is nonlinear $\left(r^{2}=0 \cdot 8164\right)$. This suggests that the polarity of the medium is not the only property of the solvent responsible for the change in rate with change in the solvent composition. The values of relative permittivity of acetic acid-water mixtures have been estimated from the corresponding values of the pure solvents ${ }^{16}$. The effect of solvent composition was analysed using the Grunwald-Winstein ${ }^{17}$ equation,

$$
\log k_{2}=m Y+\log k_{0} .
$$

A plot of $\log k_{2}$ against $Y$ is linear $\left(r^{2}=0.9996\right)$ with $m=0.58 \pm 0.03$. The value of $m$ indicates that a large charge separation takes place in the transition state of the ratedetermining step. The observed negative entropy of activation also supports this. As the charge separation takes place, the charged ends become highly solvated. This results in immobilization of a large number of solvent molecules, reflected in the loss of entropy. However, the changes in the rate constant, $k_{2}$, with the solvent composition may well be due to the changes in the acidity of the solvent. The $p \mathrm{H}$ of 1:4 $(v / v)$ acetic acid-water mixture is 1.92 , whereas that of $4: 1(\mathrm{v} / \mathrm{v})$ acetic acid-water mixture is $0 \cdot 11$. We could not study the effect of acidity on the reaction rate, as the oxidant decomposes on addition of a mineral acid.

The decrease in the value of $K$, with increase in the polarity of the medium may be attributed to the fact that when an anion reacts with a neutral molecule to form an intermediate complex, the charge is dispersed over somewhat larger areas in the product. Thus the charge density is diminished and the complex formation is facilitated by decrease in the water content of the solvent mixture. This is also in accord with the suggestion that the active oxidizing species is tribromide ion.

Hydrogen abstraction mechanism leading to free radicals may be discounted in view of the failure to induce polymerization of acrylonitrile and the fact that there is no effect of the radical scavenger on the reaction rate.

The diverse nature of the products formed in the oxidation of vicinal diols and other diols suggests that these compounds follow different mechanistic pathways. This is further supported by the two linear isokinetic plots obtained between $\log k_{2}$ at $293 \mathrm{~K}$ and at $323 \mathrm{~K}$, one for the vicinal diols and another for the rest (figure 2).

\subsection{Oxidation of vicinal diols}

Linear correlation between $\log k_{2}$ at $293 \mathrm{~K}$ and $323 \mathrm{~K}$ for the oxidation $\left(r^{2}=0.9992\right.$; slope $=0.756 \pm 0.009)$ shows that an isokinetic relationship exists in the oxidation of vicinal diols by $\mathrm{BTMAB}^{18}$. The value of the isokinetic temperature is $474 \pm 14 \mathrm{~K}$. An isokinetic relationship is a necessary condition for the validity of linear free energy relationships. It also implies that all the diols so correlated are oxidized by a similar mechanism.

Absence of a primary kinetic isotopic effect confirms that the $\alpha-\mathrm{C}-\mathrm{H}$ bond is not cleaved in the rate-determining step. In contrast, substantial primary kinetic isotopic effect was observed in the oxidation of ethanol and benzyl alcohol by BTMAB ${ }^{4}$. Thus it seems that the oxidation of monohydric alcohols and vicinal diols follows different mechanisms. 


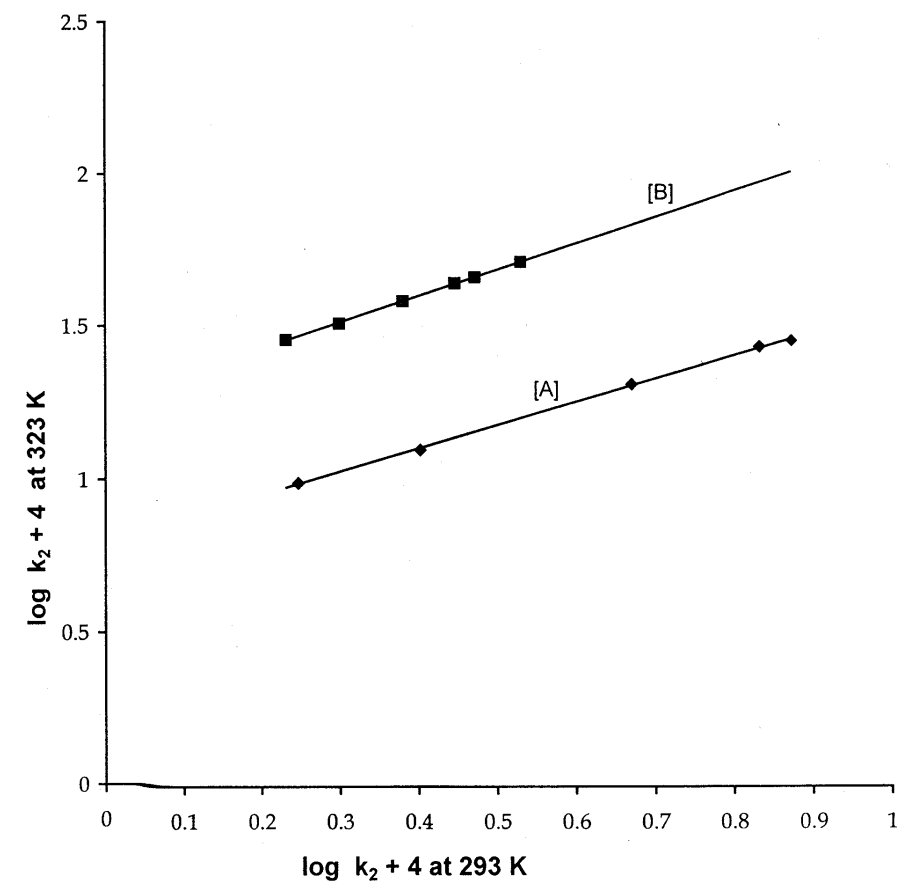

Figure 2. Isokinetic relationship in the oxidation of diols by BTMAB (A) vicinal diols, (B) other diols.

Active hydrogens like those present in the hydroxyl and carboxyl groups undergo rapid exchange in deuterium oxide. If the cleavage of the $\mathrm{O}-\mathrm{H}$ bond is involved in the rate-determining step, the $\mathrm{O}-\mathrm{H} / \mathrm{O}-\mathrm{D}$ isotope effect comes into play. In view of the observed value of the solvent isotope effect, $\mathrm{O}-\mathrm{H}$ bond cleavage in the rate-determining step is indicated. The large magnitude of the solvent isotope effect, observed in the oxidation of ethanediol, suggests that both the hydroxyl groups are involved in the ratedetermining step. The magnitude of the negative entropy of activation is more than double in the oxidation of vicinal diols as compared to that of the other compounds (cf. table 4). This points to a more rigid transition state in the oxidation of the vicinal diols. Thus, involvement of both the hydroxyl groups in the rate-determining step is indicated. The only mode of oxidation available for pinacol is fission of the bonds between the carbinol carbon atoms. That the other vicinal diols follow this mechanism is confirmed by the isolation of the products formed by the $\mathrm{C}-\mathrm{C}$ bond fission and the isokinetic relationship.

The observed Michaelis-Menten kinetics with respect to the diol led us to suggest that an intermediate complex may be formed by the interaction between the non-bonded pairs of electrons of the hydroxyl oxygens and tribromide ion in a rapid pre-equilibrium. The formation of similar complexes has also been postulated in the oxidation of aliphatic alcohols ${ }^{4 a}$ and aliphatic aldehydes ${ }^{8}$ with BTMAB as well as in the oxidation of alcohols and diols with pyridinium hydrobromide perbromide ${ }^{19,20}$. The formation of an intermediate complex is also supported by spectral evidence. Thus on the basis of all the above experimental facts, the following mechanism has been proposed (scheme 1). 


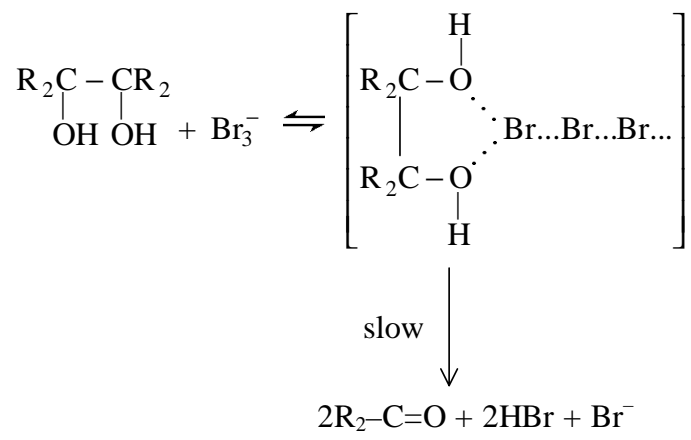

Scheme 1.

Table 8. Reaction constants of the oxidation of vicinal diols by BTMAB.

\begin{tabular}{lcccc}
\hline$T(\mathrm{~K})$ & $\rho_{I}$ & $\delta$ & $R^{2}$ & s.d.* $^{*}$ \\
\hline 293 & $-1.07 \pm 0.04$ & $-1.48 \pm 0.02$ & 0.9998 & 0.01 \\
303 & $-1.00 \pm 0.03$ & $-1.37 \pm 0.02$ & 0.9998 & 0.01 \\
313 & $-0.85 \pm 0.04$ & $-1.27 \pm 0.02$ & 0.9997 & 0.01 \\
323 & $-0.74 \pm 0.07$ & $-1.15 \pm 0.04$ & 0.9990 & 0.01 \\
\hline
\end{tabular}

*Standard deviation

The mechanism shown in scheme 1 is also supported by the observed negative entropy of activation. As charge separation takes place in the transition state of the rate-determining step, the charged ends become highly solvated. This results in the immobilization of a large number of solvent molecules, reflected in the loss of entropy.

\subsection{Correlation of structure and reactivity}

Perusal of the data in tables 3 and 4 showed that the formation constants of the dioloxidant complexes are not sensitive to the structure of the diol. However, the rate constants of the decomposition of the complexes showed considerable variation. The rate constants of decomposition were, therefore, subjected to correlation analyses.

The rate constants of the decomposition of the complexes failed to show satisfactory correlation with either the polar or the steric substituent constants separately ${ }^{21}$. Therefore, the rates were analysed in terms of the dual substituent-parameter (DSP) equation of Pavelich and Taft ${ }^{22}$,

$$
\log k=\rho_{\mathrm{I}} \Sigma \sigma_{I}+\delta \Sigma E_{s}+\log k_{0}
$$

The results, recorded in table 8, show that the rates exhibit excellent correlation with polar and steric substituent constants. Though the number of compounds (five) is rather small for a correlation analysis by the DSP equation, the correlations are excellent and the results can be used qualitatively. 
The values of the reaction constants support the proposed mechanism. The small negative polar reaction constant accords with the net flow of electrons towards the oxidant. The negative steric reaction constant implies steric acceleration of the reaction. This is probably due to increase in the steric relief on going from tetragonal $\left(s p^{3}\right)$ carbon atoms to trigonal $\left(s p^{2}\right)$ ones, with increasing substitution at the carbon atoms.

\subsection{Oxidation of other diols}

Linear correlations between values of $\log k_{2}$ at $293 \mathrm{~K}$ and $323 \mathrm{~K}\left(r^{2}=0.9996\right.$, slope $0.864 \pm 0.009$ ) for the oxidation of four non-vicinal diols, 3-methoxybutan-1-ol, and 2methoxyethanol show that all the compounds are oxidized by the same mechanism ${ }^{18}$. 3Methoxybutan-1-ol and 2-methoxyethanol are typical monohydric alcohols and, therefore, it is highly likely that these diols are oxidized by a mechanism similar to that for monohydric alcohols. The oxidation of ethanol ${ }^{4 a}$ exhibited a substantial kinetic isotopic effect confirming the cleavage of the $\alpha-\mathrm{C}-\mathrm{H}$ bond in the rate-determining step. Therefore, a mechanism, similar to the one proposed earlier ${ }^{4}$ for monohydric alcohols, accounts for the experimental results obtained in the oxidation of non-vicinal diols (scheme 2).

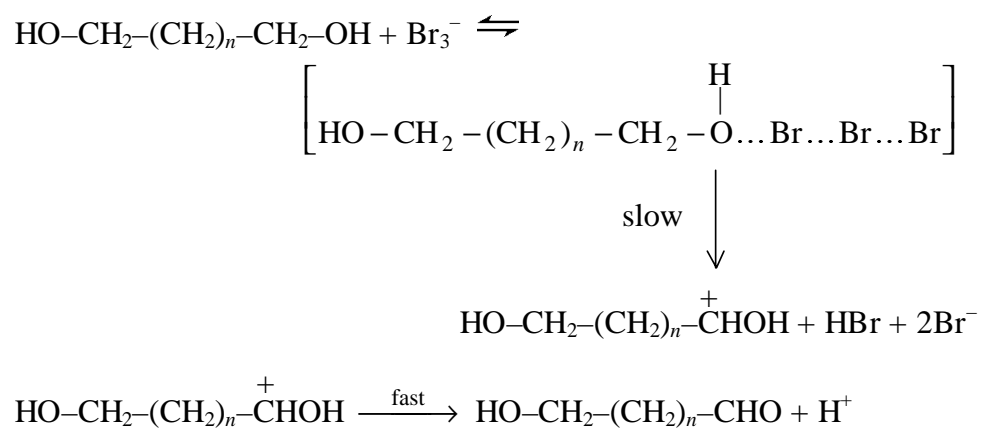

Scheme 2.

\section{Acknowledgement}

Thanks are due to the University Grants Commission, New Delhi for financial support.

\section{References}

1. Kajigaeshi S, Kakinami T, Tokiyama H, Hirakawa T and Okamoto T 1987 Bull. Chem. Soc. Jpn. 602667

2. Kajigaeshi S, Moriuaii M, Tanaka T, Fujisaki S, Kakinami T and Okamoto T 1999 J. Chem. Soc., Perkin Trans. 1897

3. Kajigaeshi S, Murakawa K, Fujisaki S and Kakinami T 1989 Bull. Chem. Soc. Jpn. 62 3376; Kajigaeshi S, Kawamukai H and Fujisaki S 1989 Bull. Chem. Soc. Jpn. 622585

4. (a) Goel S, Kothari S and Banerji K K 1996 J. Chem. Res. (S)238, (M)1318; (b) Anjana, Kothari S and Banerji K K 1999 J. Chem. Res. (S)476 (M)2118

5. Goel S, Kothari S and Banerji K K 1996 Indian J. Chem. B35 1180 
6. Goel S, Kothari S and Banerji K K 1996 J. Chem. Res. (S)510, (M)2901

7. Rao P S C, Goel S, Kothari S and Banerji K K 1998 Indian J. Chem. B37 1129

8. Goswami G, Kothari S and Banerji K K 1999 J. Chem. Res. (S)176, (M)813

9. Bunton C A 1966 Oxidation in organic chemistry (New York: Academic Press)

10. Duke F R 1947 J. Am. Chem. Soc. 693054

11. Bhatia I and Banerji K K 1983 J. Chem. Soc., Perkin Trans. 21577

12. Mathur A and Banerji K K 1987 J. Chem. Soc., Perkin Trans. 21645

13. Kemp T J and Waters W A 1963 Proc. R. Soc. A274 480

14. (a) Coffey S 1965 Rodd's chemistry of carbon compounds (Amsterdam: Elsevier) vol. 1D; (b) Rodd E H 1951 Chemistry of carbon compounds (Amsterdam: Elsevier) vol. 1A;

(c) Buckingham J et al 1982 Dictionary of organic compounds (New York: Chapman \& Hall) vol. 3; (d) West R C 1977-78 CRC handbook of chemistry and physics (CRC Press) vol. 3

15. Bradfield A E, Jones B and Orton K J P 1929 J. Chem. Soc. 2810

16. Mehnert R and Brede O 1984 Radiat. Phys. Chem. 23463

17. Falnberg A H and Winstein S 1956 J. Am. Chem. Soc. 782770

18. Exner O 1973 Prog. Phys. Org. Chem. 10411

19. Mathur D, Sharma P K and Banerji K K 1993 J. Chem. Soc., Perkin Trans. 2205

20. Mathur D, Sharma P K and Banerji K K 1995 Proc. Indian Acad. Sci. (Chem. Sci.) 107133

21. Johnson C D 1966 The Hammett equation (Cambridge: University Press) 54

22. Pavelich W A and Taft R W 1957 J. Am. Chem. Soc. 794935 